\title{
Simulation outside Magnetic Field of the Sun
}

\author{
Jianqing Wu, Zhiliang Yang* \\ Department of Astronomy, Beijing Normal University, Beijing, China \\ "E-mail:zlyang@bnu.edu.cn \\ Received May 13, 2011; revised June 15, 2011; accepted June 25, 2011
}

\begin{abstract}
We derive the viscous current in the fully ionized two-fluid plasma to generate the solar magnetic field. The global magnetic field of the Sun can be simulated by the viscous current from the differential rotation inside the Sun. The field presents a structure with 6-polar. As the viscous current is very weak, the magnetic field intensity is only about $10^{-20} \mathrm{G}$, which could be considered as the background field of the Sun. The theory is a start for the generation of solar magnetic field. The local strong magnetic field of the Sun is not considered in the paper.
\end{abstract}

Keywords: Solar Dynamo, Two-Fluid, Viscous Current

\section{Introduction}

The Sun, being a prototypical star, is 4.7 billion years old and plays a unique role in astrophysics. It allows the fundamental processes to be explored in detail since its proximity. There are various activities in the Sun, such as flares, coronal mass ejections, solar wind and magnetic storms, which directly impact on satellites, wireless communication and space weather. These activities are mainly affected by the solar magnetic field [1], so it is critical important for researching on the nature and origin of the solar magnetic field [2]. The purpose of solar dynamo theories is not only to make clear the correlation and variation among various activities, but also to explain the origin and characteristics of the magnetic field on the Sun. Studying it can promote the research of other celestial bodies [3]. Furthermore, it is important to the development of space technology and economy.

People have researched dynamo theories more than a century. In 1919, Larmor proposed that inductive phenomenon of motive fluid may explain the origin of magnetic field [4]. It opened the door of solar cycle models. Moreover, Larmor's suggestion was well consistent with the Hale's polarity law. Its main idea was that an anti-axisymmetric and equatorial axisymmetric different rotation, which pervaded the solar interior, sheared a large-scale poloidal magnetic field and produced an equatorial antiaxisymmetric toroidal field in the Sun. However, in 1934, Cowling placed a major hurdle in Larmor's path. He proved that even the ordinary purely axisymmetric magnetic flows could not sustain an axisymmetric magnetic field against Ohmic dissipation. Toroidal magnetic field and poloidal magnetic field became zero at last [5]. It was known as Cowling's anti-dynamo theorem. From then on, a lot of anti-dynamo theories emerged. They ruled out any possible case that dynamo could run in many other simplified conditions. Moreover, Bullard and Gellman found that if velocity field only had a differential rotation, there was no dynamo solution [6]. Therefore, in a very long time, people worried about whether there was still hope to make solar dynamo work.

Until 1955, there was a fundamental breakthrough. Parker proposed the spiral movement of small-scale flow [7]. This movement was caused by convection in rotating object. It could twist parts of toroidal field and make convective turbulence to rise. Then a small magnetic flow ring was formed in the radial plane. Finally, the net effect of many of these non-axisymmetric small-scale flow rings produced a large-scale radial field. At this point, it not only completed circulation of the solar dynamo, but also avoided the Cowling's anti-dynamo theorem. Ten years later, Steenbeck, Krasuse and Rädler specifically expressed out this idea using mathematical formulae [8]. It was known as mean field dynamo theory. The progress of producing large-scale magnetic field by combination of small-scale magnetic field and disturbance of velocity was called $a$-effect. Subsequently, the mean field mechanism rapidly became the mainly theoretical choice of solar dynamo models. By the late 1970's, it was reached a basic agreement about the fundamental attributes of solar dynamo, and the $a$-effect of mean field electrodynamics was at the heart of it. 
Many early solar dynamo models could reproduce several important qualitative properties of the Sun [9]. However, these models were far from perfection. In the following, we only showed four distinct directions. First, as the buoyancy effect, the magnetic field, which was strong enough to produce sunspots, could not be stored long enough to obtain sufficient amplification [10]. Second, numerical simulation of turbulent thermally-driven convection in a thick rotating spherical shell yielded magnetic field migration pattern, which appeared nothing like the observed on the Sun. Third, the solar internal differential rotation, which was detected by helioseismology, was different from that needed to produce solar-like dynamo solutions in the dynamo mechanism. It might be the most decisive. Fourth, the powers of $a$-effect and magnetic diffusivity as assumed in mean field mechanism were not the same as those which were calculated by theoretical calculation and numerical simulation.

Solar dynamo has not yet recovered from the influence of these four ways. Like all scientific crises, this situation not only provided a redesign of existing models based on the mean field mechanism, but also stimulated the exploration of new physical mechanisms of magnetic field generation. It also recovered the previous mechanisms which had been ignored in the influence of the $a$-effect. Most notably, Babcock-Leighton (BL) mechanism was firstly proposed in 1961 [11]. In late 1980's, it obtained a new development with the development of helioseismoligy. However, BL was a semi-empirical model, rather than a strict kinetic description, and it could not be self-exciting.

With both the solar different rotation inverting by helioseismology and strong magnetic field appeared in the bottom of the convective zone, the standpoint that solar dynamo was located beneath of the convective zone was further strengthened. In 1993, Parker proposed interface dynamo [12]. In this model, the two effects of the dynamo were separated in space. $a$-effect was located in the convective zone, while $\Omega$-effect was located in the tacholine zone. The success of this dynamo was that the two regions were communication by diffusive transmission of flux. Because diffusive rate was increased by turbulence, the diffusive coefficient in the tacholine zone was smaller than that in the convective zone. This model could produce strong toroidal field in the tacholine layer. It was very effective to create a magnetic field. Moreover, this region was separated from the region which produced $a$-effect. Thus, this model solved the problem of $a$ quenching effect. However, interface dynamo was very sensitive to some parameters, such as time step, spatial resolution and other numerical parameters. Slight change in these parameters might have a great impact on the result. From the model point of view, the interface dynamo was lack of robust.

Choudhuri introduced a meridional circulation to the BL model [13]. First, meridional circulation transported the magnetic flux to the polar region. Then, the magnetic flux was taken to the tacholine zone beneath of the convective zone. Third, Shearing of the poloidal magnetic field produced toroidal field by the Sun's differential rotation [14]. This was a simple BL flux transport dynamo model with meridional circulation acted as a conveyor belt [15]. Nevertheless, only the surface part of the meridional circulation was observed, the part located in the deep solar interior had not observational data. It was constructed based on the law of conservation of mass [16]. Flux transport dynamo also could not account for the detailed formative progress of cross-equatorial ring.

Solar dynamos of our discussed above were axisymmetric, but observations showed that some solar activities were non-axisymmetric. For example, magnetic activities repeated emergence at certain longitude in a time longer than one solar magnetic activity cycle. Stix proposed the first non-axisymmetric solar dynamo model [17]. Bigazzi and Ruzmaikin studied the mean field dynamo by using non-axisymmetric $a$-effect for the first time [18]. But it was so difficult to solve the equations that its development was not perfect. At present, people can only explain a few observed features of non-axisymmetry.

Large-scale solar magnetic activities were most likely explained by the magnetohydrodynamics (MHD). The main idea of the MHD was that conductive fluid cut magnetic field lines, so it produced inductive current. Then inductive current generated magnetic field. These progresses resisted energy dissipation of magnetic field caused by fluid resistance. If inductive progress was more strongly than dissipation progress, the MHD dynamo could run continuously. Some complex physical processes could be described by a set of differential equations in the MHD dynamo. Therefore, it was possible to research the nature of large-scale of solar various aspects by simulating the MHD dynamo. This approach had been used successfully in the earth dynamo [19]. As solar large-scale and extreme parameter regions, it will be a long process for us to simulate the entire solar magnetic field. However, with the development of computer technology, more and more scientists believed that the prospect of MHD dynamo is optimistic [20,21].

At the beginning of this paper, we outline the development of solar dynamos. The problem of Ohm's law in fully ionized two-fluid is analyzed in Section 2. In Section 3, the viscous current is derived in detail in the fully ionized two-fluid plasma, and we obtain the magnetic field vector and magnetic field lines maps. Section 4 is the discussion of the results and outlook.

\section{Problem of Generalized Ohm's Law}

Magnetic field is produced by highly ionized plasma on 
the Sun. Traditionally, conductive fluid $\boldsymbol{V}$ cuts magnetic field $\boldsymbol{B}$ to produce inductive electric field $\boldsymbol{V} \times \boldsymbol{B}$, and there will be generated current from generalized Ohm's law

$$
\boldsymbol{J}=\sigma(E+\boldsymbol{V} \times \boldsymbol{B})
$$

The current will produce magnetic field based on Ampere's law $\nabla \times \boldsymbol{B}=\mu_{0} \boldsymbol{J}$, then the magnetic field can produce current in accordance with Faraday's law. Lorentz force is $\boldsymbol{J} \times \boldsymbol{B}$, which is caused by the interaction of magnetic field and current, resists against the force of exciting movement. Thus the interaction of these progresses can maintain a self-generation field. Using Maxwell's equations [22], we can obtain magnetic inductive equation

$$
\frac{\partial \boldsymbol{B}}{\partial t}=\nabla \times(\boldsymbol{V} \times \boldsymbol{B})+\eta \nabla^{2} \boldsymbol{B}
$$

where $\eta$ is magnetic diffusivity.

Many dynamo models depend on mean field mechanism [23]. For simplicity, the idea of mean field mechanism is to separate the magnetic field $\boldsymbol{B}$ and the velocity $\boldsymbol{V}$ into mean and fluctuant components, then use suitable average procedure, so that

$$
\boldsymbol{B}=\langle\boldsymbol{B}\rangle+\boldsymbol{b}, \boldsymbol{V}=\langle\boldsymbol{V}\rangle+\boldsymbol{v}
$$

Where $\langle\boldsymbol{B}\rangle$ is the mean magnetic field component. $\boldsymbol{b}$ is the fluctuant component and the average value of $b$ is $\langle\boldsymbol{b}\rangle=0 .\langle\boldsymbol{V}\rangle$ is the mean velocity and $\langle\boldsymbol{V}\rangle=0$. Then the average inductive equation takes the form

$$
\frac{\partial}{\partial t}\langle\boldsymbol{B}\rangle=\nabla \times(\langle\boldsymbol{V}\rangle \times\langle\boldsymbol{B}\rangle+\alpha\langle\boldsymbol{B}\rangle+(\eta+\beta) \nabla \times\langle\boldsymbol{B}\rangle)
$$

where $\quad \alpha=-\frac{1}{3} \int_{0}^{\infty}\left\langle\boldsymbol{v}(t) \cdot \nabla \times v\left(t-t^{\prime}\right)\right\rangle \mathrm{d} t^{\prime}$, and $\beta=-\frac{1}{3}$ $\int_{0}^{\infty}\left\langle v(t) \cdot v\left(t-t^{\prime}\right)\right\rangle \mathrm{d} t^{\prime} \quad$ [24]. Comparison of Equations and (4), we can see that there are two changes. First, diffusion coefficient is increased by $\beta$, another more important change is adding the item $\alpha\langle\boldsymbol{B}\rangle$, which is a guarantee that mean field $\langle\boldsymbol{B}\rangle$ constraints from the antidynamo theorem. Usually, the key item of $\alpha\langle\boldsymbol{B}\rangle$ in mean field inductive Equation (4) is called $\alpha$-effect. We expect that $\beta \gg \eta$, though molecular diffusion is none the less an essential part of the dynamo process. In fact, the $\alpha$-effect depends on the presence of 'gyrotropic' turbulence with a net kinetic helicity $H=\langle\boldsymbol{v} . \nabla \times \boldsymbol{v}\rangle$ in the small-scale motion.

The mean field inductive Equation (4) is the starting point for most researchers of solar dynamo theories. It is also used by the scholars, who study the Earth or stars dynamos. But we must remember that this set of approximations can only be justified if there is indeed a separation of scales or either the magnetic Reynolds number $R_{m} \ll 1$ or $\tau \ll l / u$. Neither of the latter con- ditions is valid in the Sun.

From Equations (2) and (4), we can see that both the first items of right side of these equations came from the generalized Ohm's law, which is Equation (1). The law is right for a single species of charged particles, but it is not suitable for fully ionized two-fluid plasma.

When a charged particle runs in the static electromagnetic field, we can easily obtain its dynamic equation. A particle with charge $q$ will run in the following dynamic equation in the electromagnetic field

$$
m \frac{\mathrm{d} \boldsymbol{V}}{\mathrm{d} t}=q \boldsymbol{E}+q(\boldsymbol{V} \times \boldsymbol{B})
$$

The particle will run in helical line. If $\boldsymbol{E}$ is perpendicular to $\boldsymbol{B}$, we can obtain a drift velocity $\boldsymbol{V}_{d}=\frac{\boldsymbol{E} \times \boldsymbol{B}}{B^{2}}$ [25].

We can divide the electric field into two parts. $E_{\|}$is parallel to the magnetic field and $E_{\perp}$ is perpendicular to the magnetic field. The particle runs freely in the direction of magnetic field. While the particle runs in sinusoid and its average motion is zero in the direction of electric field $E_{\perp}$. The particle not only has Larmor motion, but also has a drift velocity $\boldsymbol{V}_{d}$, which is perpendicular to both electric field and magnetic field. If there are only electrons and ions in the neutral fully ionized plasma, there are not considered collisions between electrons and ions and the actions of external forces. The drift velocity is independent of mass and charge of particle, so both the electrons and ions have the same drift velocity in the same direction and it will not produce current in the drift direction, which is perpendicular to both electric field $\boldsymbol{E}$ and magnetic field $\boldsymbol{B}$. As we know, $\boldsymbol{V} \times \boldsymbol{B}$ is always perpendicular to magnetic field, so the current from $\boldsymbol{V} \times \boldsymbol{B}$ is zero. The current only appears in the direction of magnetic field without other interaction. Therefore, Equation (1) is not suitable for the particle in the above condition and we also think it is not suitable for deriving the dynamo models.

\section{Magnetic Field Outside the Sun Using Viscous Current}

Astrophysicists try to explain the magnetic field of various objects using dynamo theories. Unfortunately, dynamo theories can not completely explain all the phenomena. Particles current, which can generate magnetic field, plays a very important role in the study of dynamo theories. If we ignore the impact on the magnetic field of particles, it will prevent us from better understanding some important aspects of plasma physics properties in universe.

\subsection{Two-Fluid Model of Plasma}

The single fluid theory of MHD is the simplest way to 
describe plasma [26]. The complete model may be the full particle simulation. To understand the physical process, MHD gives a good description of some waves in plasma. However, it is not perfect enough. The one-fluid MHD model always treats the plasma as a whole and not considers the interior particles respectively, which are accepted in the treatment of a few phenomena. Moreover, based on this model we can not understand the physical process of current generation. There are still a number of phenomena that can not be solved in this way, such as transferring energy from one region to another, formation of double layers and the occurrence of explosive events. Therefore, we need to consider from another aspect, which is not from the field but from the particle aspect. It is obvious that the velocity difference between particles can arouse the generation of current. Here we call it the two-fluid model. In the following, we will assume the plasma only consist of ions and electrons.

The plasma fluid equations with viscous current are given by [27]

$$
\begin{gathered}
\frac{\partial n_{\alpha}}{\partial t}+\nabla \cdot\left(n_{\alpha} \boldsymbol{V}_{\alpha}\right)=0 \\
\frac{\mathrm{d} \boldsymbol{V}_{\alpha}}{\mathrm{d} t}=\frac{q_{\alpha}}{m_{\alpha}}\left(\boldsymbol{E}+\frac{1}{c} \boldsymbol{V}_{\alpha} \times \boldsymbol{B}\right)-v_{\alpha \beta}\left(\boldsymbol{V}_{\alpha}-\boldsymbol{V}_{\beta}\right) \\
-\frac{\nabla P_{\alpha}}{n_{\alpha} m_{\alpha}}-\frac{\mu_{\alpha} \nabla^{2} \boldsymbol{V}_{\alpha}}{n_{\alpha} m_{\alpha}}
\end{gathered}
$$

where $\boldsymbol{V}_{\alpha}$ and $\boldsymbol{V}_{\beta}$ are the velocities of ions or electrons, $v_{\alpha \beta}$ is the collision frequency between ions and $\mu_{\alpha}$

electrons, $P_{\alpha}$ is the viscous coefficient of ions or elec$n$

trons. The charge of ion is assumed $q_{\alpha}=e$ and that of electron is assumed $q_{e}=-e$ in the ions and electrons plasma. Here, $e$ is a positive unit charge constant. $n_{\alpha}$ is the plasma density, $m_{\alpha}$ is the mass of ions or electrons and $P_{\alpha}$ is the pressure of ions and electrons. The ion momentum transfer equation

$$
\begin{aligned}
& \frac{\mathrm{d} \boldsymbol{V}_{i}}{\mathrm{~d} t}=\frac{e}{m_{i}}\left(\boldsymbol{E}+\frac{1}{c} \boldsymbol{V}_{i} \times \boldsymbol{B}\right)-v_{i e}\left(\boldsymbol{V}_{i}-\boldsymbol{V}_{e}\right) \\
& -\frac{\nabla P_{i}}{n_{i} m_{i}}-\frac{\mu_{i} \nabla^{2} \boldsymbol{V}_{i}}{n_{i} m_{i}}
\end{aligned}
$$

and the electron momentum transfer equation

$$
\begin{aligned}
& \frac{\mathrm{d} \boldsymbol{V}_{e}}{\mathrm{~d} t}=\frac{-e}{m_{e}}\left(\boldsymbol{E}+\frac{1}{c} \boldsymbol{V}_{e} \times \boldsymbol{B}\right)-v_{e i}\left(\boldsymbol{V}_{e}-\boldsymbol{V}_{i}\right) \\
& -\frac{\nabla P_{e}}{n_{e} m_{e}}-\frac{\mu_{e} \nabla^{2} \boldsymbol{V}_{e}}{n_{e} m_{e}}
\end{aligned}
$$

In the peusi-neutral plasma, $n_{i}=n_{e}=n$, where $n_{i}$ and $n_{e}$ are the densities of ions and electrons respectively. $n$ is the plasma density. We use $\boldsymbol{j}=n_{i} e\left(\boldsymbol{V}_{i}-\boldsymbol{V}_{e}\right)$ as the current density.

Since the mass of ion is much larger than that of electron, and all quantities in the differential of time are supposed to be ignored in the steady case, we can get the following equation from Equations (8) and (9)

$$
\begin{aligned}
& \boldsymbol{j}=\frac{c^{2}}{4 \pi \eta}\left[\frac{1}{c}\left(\boldsymbol{V}_{i} \times \boldsymbol{B}\right)+\frac{1}{n e c}(\boldsymbol{j} \times \boldsymbol{B})+\frac{1}{n e} \nabla P_{e}\right. \\
& \left.-\frac{m_{e}}{n e m_{i}} \mu_{i} \nabla^{2} \boldsymbol{V}_{i}+\frac{1}{n e} \mu_{e} \nabla^{2} \boldsymbol{V}_{e}\right]
\end{aligned}
$$

where $\eta=\frac{c^{2}}{4 \pi \sigma}$ is the resistivity, $\sigma=\frac{n e^{2}}{m_{e} v_{e i}}$ is the conductivity. The first term $\frac{1}{c}\left(\boldsymbol{V}_{i} \times \boldsymbol{B}\right)$ is the inductive current with plasma moved in magnetic field, the second term $\frac{1}{n e c}(\boldsymbol{j} \times \boldsymbol{B})$ is the Hall current, the third term $\frac{1}{n e} \nabla P_{e}$ is the Bierman's battery and the last two items $\left(-\frac{m_{e}}{n e m_{i}} \mu_{i} \nabla^{2} \boldsymbol{V}_{i}+\frac{1}{n e} \mu_{e} \nabla^{2} \boldsymbol{V}_{e}\right)$ are the current due to the viscosity of ions and electrons.

The Hall current and Bierman's battery are commonly accepted and studied in laboratory plasma physics. However, the current from viscosity of ions and electrons is not mentioned in many literatures.

In physics, external force $\boldsymbol{F}$ acting on charged particle with charge $\boldsymbol{Q}$ could be equivalent to an electrical force. The equivalent electrical field is $\boldsymbol{E}=\boldsymbol{F} / \boldsymbol{Q}$ for charged fluid (ions or electrons). The charge density is $n e$ and the force in unit volume is $\boldsymbol{f}$, then we can get the electric field $\boldsymbol{E}=\boldsymbol{f} / n e$. If the fluid has electrical resistivit $\eta$, we can get the electrical current $\boldsymbol{j}=\frac{c^{2}}{4 \pi \eta} \boldsymbol{E}=\frac{c^{2}}{4 \pi \eta} \frac{\boldsymbol{f}}{n e}$. Therefore, viscous current of ions and electrons is

$$
\boldsymbol{j}=\frac{c^{2}}{4 \pi \eta}\left(-\frac{m_{e}}{n e m_{i}} \mu_{i} \nabla^{2} \boldsymbol{V}_{i}+\frac{1}{n e} \mu_{e} \nabla^{2} \boldsymbol{V}_{e}\right)
$$

The viscous coefficients of ions and electrons have the following forms respectively [28-30]

$$
\begin{aligned}
& \mu_{i}=2.21 \times 10^{-15} \frac{T_{i}^{5 / 2} A_{i}^{1 / 2}}{\ln \Lambda}\left(\mathrm{g} \cdot \mathrm{cm}^{-1} \cdot \mathrm{s}^{-1}\right) \\
& \text { and } \\
& \mu_{e}=2.21 \times 10^{-15} \frac{T_{e}^{5 / 2} A_{e}^{1 / 2}}{\ln \Lambda}\left(\mathrm{g} \cdot \mathrm{cm}^{-1} \cdot \mathrm{s}^{-1}\right)
\end{aligned}
$$


where $A_{i}$ and $A_{e}$ are the atomic weight of ions and electrons, $A_{i}=1$ and $A_{e}=1 / 1836 . T_{i}$ and $T_{e}$ are the temperatures of ions and electrons in the unit of $K$, $\Lambda=\overline{\lambda_{D} / r_{0}}$ [25], $r_{0}$ is an impact parameter and $\lambda_{D}$ is the largest impact parameter.

Substituting the electrical resistivity with the collision frequency between ions and electrons, we can get

$$
\boldsymbol{j}=-\frac{e \mu_{i}}{m_{i} v_{e i}} \nabla^{2} \boldsymbol{V}_{i}+\frac{e \mu_{e}}{m_{e} v_{e i}} \nabla^{2} \boldsymbol{V}_{e}
$$

If the ions and electrons have the same second-order differential velocities $\nabla^{2} \boldsymbol{V}_{i}=\nabla^{2} \boldsymbol{V}_{e}=\nabla^{2} \boldsymbol{V}, \boldsymbol{V}$ is the plasma velocity, and the temperature of electrons is higher than or equal to that of ions in general case. Viscous current of ions can be ignored, so

$$
\mathbf{j}=\frac{e \mu_{e}}{m_{e} v_{e i}} \nabla^{2} \boldsymbol{V}
$$

where the collision frequency $v_{e i}=\frac{n}{1.52 \times T_{e}^{3 / 2}}\left(s^{-1}\right)$,

then we get

$$
\boldsymbol{j}=\frac{1.37 \times 10^{-8} T_{e}^{4}}{n \ln \Lambda} \nabla^{2} \boldsymbol{V}\left(\mathrm{A} \cdot \mathrm{cm}^{-2}\right),
$$

where $n$ is the plasma density in the unit of $\mathrm{cm}^{-3}$ and $\boldsymbol{V}$ is the plasma velocity in the unit of $\mathrm{cms}^{-1}$.

From Equation (16), we can see the viscous current depends strongly on the temperature of electrons, as well as the plasma density and the second-order differential velocity.

\subsection{Differential Rotation}

The differential rotation is commonly found in most astrophysical objects with the advent of helioseismology. Solar different rotation inverting by helioseismology has shown that the strongest shear is associated with the so-called tachocline [31,32], the rotation shear layer located immediately beneath the core-envelope interface. The result can be used to calculate the viscous current inside the Sun.

The differential rotation $\Omega$ can be expressed as following [33,34],

$$
\Omega=\Omega_{c}+\frac{1}{2}\left\{1+\operatorname{erf}\left[\frac{2\left(r-r_{c}\right)}{d_{1}}\right]\right\}\left(\Omega_{s}-\Omega_{c}\right)
$$

where $\Omega_{s}=\Omega_{e q}+a_{2} \cos ^{2} \theta+a_{4} \cos ^{4} \theta$ is the surface latitude differential rotation and $\operatorname{erf}(x)$ is the error function. The other parametric values are set as $\Omega_{\mathrm{c}} / 2 \pi=432.8 n \mathrm{~Hz}$, $\Omega_{e q} / 2 \pi=460.7 n \mathrm{~Hz}, a_{2}=-62.69 n \mathrm{~Hz}, a_{4}=-67.13 n \mathrm{~Hz}$, $r_{c}=0.7 R_{s}$ and $d_{1}=0.05 R_{s}$. This describes a solar-like differential rotation profile. Moreover, it has purely latitudinal differential rotation $\Omega_{s}$ with equatorial acceleration in the "envelope" and smoothes matching across a "tacholine" of thickness $d_{1}$ on a "core" rotating rigidly at a rate $\Omega_{c} . r_{c}$ is the central radius of the tachocline, where the radial shear is confined. The differential rotation profile resembles rather closely that derived from fully two-dimensional helioseismic inversion $[35,36] . \quad R_{s}$ is the solar radius and is set to be $7 \times 10^{10} \mathrm{~cm}$.

The electron temperature is about $T_{e}=2 \times 10^{6} \mathrm{~K}$ around $0.75 R_{s}$, and electron density is $n_{e}=245 N_{A} \exp$ $\left(-10.54 r / R_{s}\right) \mathrm{cm}^{-3} . N_{A}$ is an Avogadro's number [37, 38].

\subsection{Viscous Current}

If we want to obtain the viscous current $\boldsymbol{j}$, we must calculate $\nabla^{2} \boldsymbol{V}$ from Equation (16) at first. Because the Sun is a sphere, we expand it in the spherical coordinate

$$
\begin{aligned}
\nabla^{2} \boldsymbol{V} & =\left[\nabla^{2} V_{r}-\frac{2}{r^{2}}\left(V_{r}+\cot \theta V_{\theta}+\frac{\partial V_{\theta}}{\partial \theta}+\frac{1}{\sin \theta} \frac{\partial V_{\varphi}}{\partial \varphi}\right)\right] \boldsymbol{e}_{r} \\
& +\left[\nabla^{2} V_{\theta}+\frac{2}{r^{2}}\left(\frac{\partial V_{r}}{\partial \theta}-\frac{V_{\theta}}{2 r^{2} \sin ^{2} \theta}-\frac{\cos \theta}{\sin ^{2} \theta} \frac{\partial V_{\varphi}}{\partial \varphi}\right)\right] \boldsymbol{e}_{\theta} \\
& +\left[\nabla^{2} V_{\varphi}+\frac{2}{r^{2}}\left(\frac{1}{\sin \theta} \frac{\partial V_{r}}{\partial \varphi}-\frac{V_{\varphi}}{2 \sin ^{2} \theta}+\frac{\cos \theta}{\sin ^{2} \theta} \frac{\partial V_{\theta}}{\partial \varphi}\right)\right] \boldsymbol{e}_{\varphi}
\end{aligned}
$$

The direction of $\Omega$ is along the rotation axis, $r$ is point to outside and velocity is $\boldsymbol{V}=\Omega \times \boldsymbol{r}$, thus its direction is $\boldsymbol{e}_{\varphi}$. That is to say $\boldsymbol{V}$ only has the $\boldsymbol{e}_{\varphi}$ component. Equation (18) can be simplified

$$
\nabla^{2} \boldsymbol{V}=\left(\nabla^{2} V_{\varphi}-\frac{V_{\varphi}}{r^{2} \sin ^{2} \theta}\right) \boldsymbol{e}_{\varphi}
$$

There is an item $\nabla^{2} \boldsymbol{V}_{\varphi}$ in the Equation (19) and then we expand it in the spherical coordinate. We obtain its form as following

$$
\begin{aligned}
& \nabla^{2} V_{\varphi}=\frac{1}{r^{2}} \frac{\partial}{\partial r}\left(r^{2} \frac{\partial V_{\varphi}}{\partial r}\right)+\frac{1}{r^{2} \sin \theta} \frac{\partial}{\partial \theta}\left(\sin \theta \frac{\partial V_{\varphi}}{\partial \theta}\right) \\
& +\frac{1}{r^{2} \sin ^{2} \theta} \frac{\partial^{2} V_{\varphi}}{\partial \varphi^{2}}
\end{aligned}
$$

Since $V_{\varphi}$ is symmetric about $e_{\varphi}$, it means $\frac{\partial V_{\varphi}}{\partial \varphi}=0$. So $\nabla^{2} V_{\varphi}$ can be written as

$$
\nabla^{2} V_{\varphi}=\frac{1}{r^{2}} \frac{\partial}{\partial r}\left(r^{2} \frac{\partial V_{\varphi}}{\partial r}\right)+\frac{1}{r^{2} \sin \theta} \frac{\partial}{\partial \theta}\left(\sin \theta \frac{\partial V_{\varphi}}{\partial \theta}\right)
$$

Substituting Equation (21) into Equation (19), we can obtain

$$
\begin{aligned}
\nabla^{2} \boldsymbol{V} & =\left[\frac{1}{r^{2}} \frac{\partial}{\partial r}\left(r^{2} \frac{\partial V_{\varphi}}{\partial r}\right)+\frac{1}{r^{2} \sin \theta} \frac{\partial}{\partial \theta}\left(\sin \theta \frac{\partial V_{\varphi}}{\partial \theta}\right)\right. \\
& \left.-\frac{V_{\varphi}}{r^{2} \sin ^{2} \theta}\right] \boldsymbol{e}_{\varphi}
\end{aligned}
$$


From Equation (22), we can see that if we want to obtain the value of $\nabla^{2} \boldsymbol{V}$, we must calculate the three items on the right of the equation. Substituting $V_{\varphi}=\Omega \cdot r$ into Equation (22), the first item can be expressed

$$
\begin{aligned}
& \frac{1}{r^{2}} \frac{\partial}{\partial r}\left(r^{2} \frac{\partial V_{\varphi}}{\partial r}\right)=\frac{1}{r}\left\{2 \Omega_{c}+\left(\Omega_{s}-\Omega_{c}\right)\right. \\
& \left.\left[1+\operatorname{erf}(x)+\left(8-\frac{r x}{d_{1}}\right) \frac{r}{\sqrt{\pi} d_{1}} e^{(-x)^{2}}\right]\right\}
\end{aligned}
$$

where $x=\frac{2\left(r-r_{c}\right)}{d_{1}}$. The second item can be written

$$
\begin{aligned}
& \frac{1}{r^{2} \sin \theta} \frac{\partial}{\partial \theta}\left(\sin \theta \frac{\partial V_{\varphi}}{\partial \theta}\right)=\frac{A}{r}\left[2 a_{2} \sin ^{2} \theta\left(1+6 \cos ^{2} \theta\right)\right. \\
& \left.-4 \cos ^{2} \theta\left(a_{2}+2 a_{4} \cos ^{2} \theta\right)\right]
\end{aligned}
$$

and the third item can be expressed

$$
\frac{V_{\varphi}}{r^{2} \sin ^{2} \theta}=\frac{\Omega}{r \sin ^{2} \theta}
$$

where $A=\frac{1}{2}\left\{1+\operatorname{erf}\left[\frac{2\left(r-r_{c}\right)}{d_{1}}\right]\right\}$.

Now, we have calculated the $\nabla^{2} \boldsymbol{V}$, which is the function of $r$ and $\theta$. We substitute it into the Equation (16). Then we will obtain the distribution of viscous current in the Sun.

\subsection{Magnetic Field outside the Sun}

We have obtained the viscous current and then we begin to calculate the outside magnetic field of the Sun. The distant formula from inner to exterior of the Sun is

$$
\left|\boldsymbol{r}-\boldsymbol{r}^{\prime}\right|=\sqrt{\left(\boldsymbol{x}-\boldsymbol{x}^{\prime}\right)^{2}+\left(\boldsymbol{y}-\boldsymbol{y}^{\prime}\right)^{2}+(\mathbf{z}-\mathbf{z})^{2}}
$$

Then we use Biot-Savart law to compute external magnetic field of the Sun

$$
\boldsymbol{B}(\boldsymbol{r})=\frac{1}{c} \int_{v^{\prime}} \frac{\boldsymbol{J}\left(\boldsymbol{r}^{\prime}\right) \times\left(\boldsymbol{r}-\boldsymbol{r}^{\prime}\right)}{\left|\boldsymbol{r}-\boldsymbol{r}^{\prime}\right|^{3}} \mathrm{~d} v^{\prime}
$$

where $\mathrm{c}$ is a proportional coefficient, its value is $3 \times 10^{10}$. $\boldsymbol{r}^{\prime}$ is the inner radius of the Sun, $\boldsymbol{r}$ is the external radius, $d v^{\prime}$ is the inner differential volume element and $v^{\prime}$ is the entire volume inside the Sun. In order to obtain the value of $\boldsymbol{B}(\boldsymbol{r})$, we divide it into $B(x), B(y)$ and $B(z)$ components in Cartesian coordinate. Therefore, we have three components of solar magnetic field

$$
B(x)=\frac{1}{c} \int_{v^{\prime}} \frac{J_{y} z_{0}}{\left|r-r^{\prime}\right|^{3}} \mathrm{~d} v^{\prime}
$$

$$
\begin{gathered}
B(y)=\frac{1}{c} \int_{v^{\prime}} \frac{-J_{x} z_{0}}{\left|r-\mathbf{r}^{\prime}\right|^{3}} \mathrm{~d} v^{\prime} \\
B(z)=\frac{1}{c} \int_{v^{\prime}} \frac{J_{x} y_{0}-J_{y} x_{0}}{\left|r-r^{\prime}\right|^{3}} \mathrm{~d} v^{\prime}
\end{gathered}
$$

Now, we have derived the formulae of magnetic field outside the Sun. Using Equations (28), (29) and (30), we can obtain any point value of magnetic field outside the Sun.

\subsection{Magnetic Field Maps}

Figure 1 is the magnetic field vector map outside the Sun. It describes the directional change of magnetic field. We integrate the magnetic field $\boldsymbol{B}$ from $R_{s}$ to $3 R_{S}$.

Figure 2 is a map of magnetic field lines outside the Sun. It is very apparent that some of the magnetic field lines are open, and the others are closed. The north and south poles are negative and positive respectively. We can see that the magnetic field outside the Sun is symmetric about the solar axis and anti-axisymmetric about the equator. We find that there are six closed areas of magnetic field lines outside the Sun. Moreover, there are some cross-equatorial rings through the equator. The two small butterfly-shaped magnetic field lines above point to the north pole. However, the change happens approximately at the latitude $20^{\circ}$, which magnetic field lines extend outward directly. When the latitude is more than $20^{\circ}$, magnetic field lines extend outward and gradually point to the north pole. While the latitude is less than $20^{\circ}$, magnetic field lines extend toward the equator. With the extension of the magnetic field lines, they don't stop at the equator, but across the equator and continue to extend to the southern hemisphere, until they reach the points, the absolute values of which are the same as they started points. These magnetic field lines, crossed the equator, are called as cross-equatorial rings. The two areas of magnetic field lines below the Sun come out from South Pole. Some of them also become open, and the others return to the Sun's surface after extending out some distance.

The magnetic field of Figure 1 and Figure 2 is generated by viscous current in Equation (16). But the viscous current is very weak, thus the magnetic field $\boldsymbol{B}$ derived from it is very weak. The magnitude of the magnetic field strength is only about $10^{-20} \mathrm{G}$. It could be the background magnetic field of the Sun.

\section{Conclusions}

People have constructed a variety of solar dynamos to explain the cycle activities of the Sun, the origin and gen- 
eration of the magnetic field. However, these dynamos have their own shortcomings, and can not explain fully all aspects of the Sun.

Ions and electrons velocities are identical in fully ionized two-fluid plasma without considering the effect of collisions and external forces, so there is no current in the plane which is perpendicular to the magnetic field. The current only appears in the direction which is parallel to the magnetic field. Thus generalized Ohm's law does not fit in this condition.

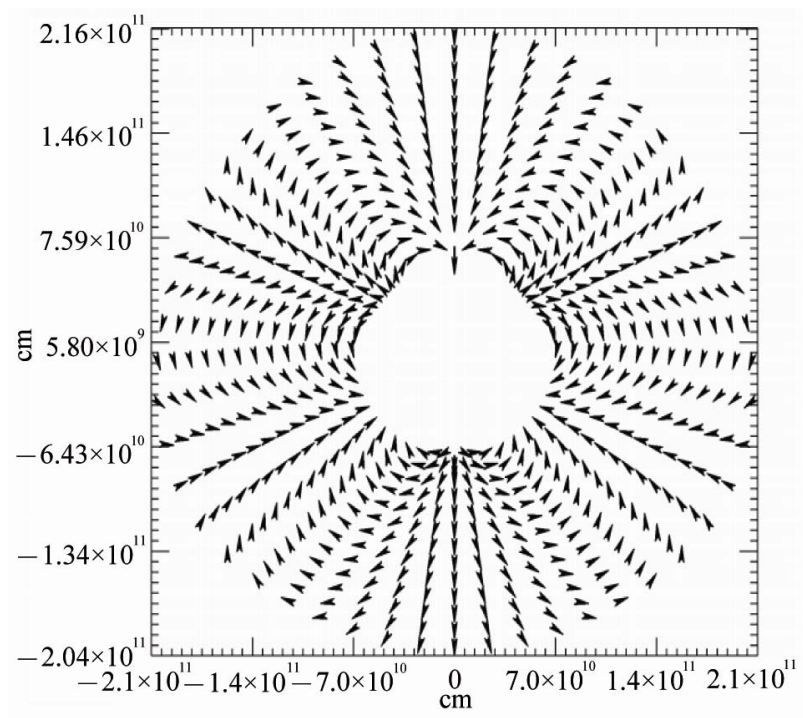

Figure 1. It is the magnetic field vector figure outside the Sun. The integral scale is form $R_{s}$ to $3 R_{s}$.

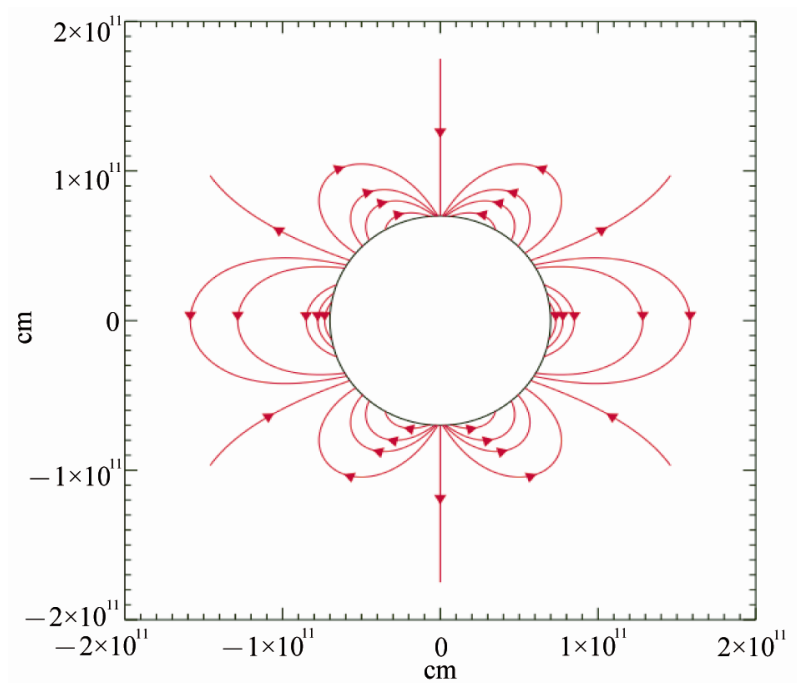

Figure 2. It is a figure of magnetic field lines outside the Sun. Some of the magnetic field lines are open, and the others are closed. The north pole is negative polarity and the south pole is positive polarity. There are six closed magnetic field lines areas outside the Sun. Furthermore, there are some cross-equatorial rings through the equator.
In fully ionized two-fluid plasma, viscous current strongly depends on the electron temperature, plasma density and second-order differential velocity. The viscous current may be an important component in solar chromosphere and corona since high electron temperature.

We can obtain the specific viscous current formula using differential rotation. Based on Biot-Savart law, we can calculate any point value of magnetic field outside the Sun through the viscous current. Then we get the figures of solar vector magnetic and magnetic field lines. We plot the magnetic field of the Sun based on the differential motion and the parameters of solar model. The magnetic field of the Sun is axis-symmetric. It could be the background magnetic field of the Sun. As viscous current is very weak, the magnetic field is also very weak. It is on the order of $10^{-20} \mathrm{G}$. So far, people have observed the local magnetic field of the Sun. Because the whole magnetic field of the Sun is very weak, it is very different from observation. It is also our future work. Since the viscosity of the electrons depends sensitively on the temperature of the electrons, we expect a reasonable electron temperature from observation or theoretical model. Although the magnetic field is very weak, this is the first time we tentatively calculate the magnetic field outside the Sun with viscous current. Most importantly, we obtain the maps of vector magnetic field and magnetic field lines outside the Sun.

In the near future, we will gradually consider external forces, partial ionized plasma and three-fluid, which contribute to the outside magnetic field of the Sun. It would be a very interesting exploration [39].

\section{References}

[1] M. Ossendrijver, "The Solar Dynamo," The Astronomy and Astrophysics Review, Vol. 11, No. 4, 2003, pp. 287-367. doi:10.1007/s00159-003-0019-3

[2] P. Charbonneau, "Dynamo Models of the Solar Cycle," 2005. http://www.livingreviews.org/lrsp-2010-3

[3] S. M. Tobias, "The Solar Dynamo," Philosophical Transactions of the Royal Society A, Vol. 360, No. 1801, 2002, pp. 2741-2756. doi:10.1098/rsta.2002.1090

[4] S. J. Larmor, "The Relativity of the Forces of Nature II," Monthly Notices of the Royal Astronomical Society, Vol. 80, No. 1, 1919, pp. 118-138.

[5] T. G. Cowling, "The Stability of Gaseous Stars," Monthly Notices of the Royal Astronomical Society, Vol. 94, 1934, pp. 768-782.

[6] E. Bullard and H. Gellman, "Homogeneous Dynamos and Terrestrial Magnetism," Philosophical Transactions of the Royal Society A, Vol. 247, No. 928, 1954, pp. 213278. doi:10.1098/rsta.1954.0018

[7] E. N. Parker, "The Formation of Sunspots from the Solar Toroidal Field," Astrophysical Journal, Vol. 121, 1955, pp. 491-507. doi:10.1086/146010 
[8] M. Steenbeck, K. Krause and K. H. Rädler, "A Calculation of the Mean Electromotive Force in an Electrically Conducting Fluid in Turbulent Motion, under the Influence of Coriolis Forces," Zeitschrift Naturforschung Teil A, Vol. 21, 1966, pp. 369-375.

[9] M. Stix, "Differential Rotation and the Solar Dynamo," Astronomy \& Astrophysics, Vol. 47, No. 2, 1976, pp. 243-254.

[10] E. N. Parker, "Cosmical Magnetic Fields: Their Origin and Their Activity," Oxford University Press, New York, 1979, pp. 40-90.

[11] H. W. Babcock, "The Topology of the Sun's Magnetic Field and the 22-Year Cycle," Astrophysical Journal, Vol. 133, 1961, pp. 572-587. doi:10.1086/147060

[12] E. N. Parker, "A Solar Dynamo Surface Wave at the Interface between Convection and Nonuniform Rotation," Astrophysical Journal, Vol. 408, No. 2, 1993, pp. 707719. doi: $10.1086 / 172631$

[13] A. R. Choudhuri, M. Schüssler and M. Dikpati, "The Solar Dynamo with Meridional Circulation," Astronomy \& Astrophysics, Vol. 303, No. 2, 1995, pp. 29-32.

[14] M. Dikpati and G. Giloman, "Global Solar Dynamo Models: Simulations and Predictions," Journal of Astrophysics and Astronomy, Vol. 29, No. 1-2, 2008, pp. 29-39. doi:10.1007/s12036-008-0004-3

[15] M. Dikpati and P. A. Gilman, "Flux-Transport Solar Dynamos," Space Science Reviews, Vol. 144, No. 1-4, 2009 , pp. 67-75. doi:10.1007/s11214-008-9484-3

[16] D. Nandy and A. R. Choudhuri, "Explaining the Latitudinal Distribution of Sunspots with Deep Meridional Flow," Science, Vol. 296, No. 5573, 2002, pp. 1671-1673. doi:10.1126/science.1070955

[17] M. Stix, “A Non-Axisymmetric $\alpha$-Effect Dynamo," Astronomy \& Astrophysics, Vol. 13, 1971, pp. 203-208.

[18] A. Bigazzi and A. Ruzmaikin, "The Sun's Preferred Longitudes and the Coupling of Magnetic Dynamo Modes," Astronomical Journal, Vol. 604, No. 2, 2004, pp. 944959.

[19] G. A. Glatzmaier and P. H. Roberts, "A Three-Dimensional Self-Consistent Computer Simulation of a Geomagnetic Field Reversal," Nature, Vol. 377, 1995, pp. 203-209. doi:10.1038/377203a0

[20] V. Archontis, K.Tsinganos and C. Gontikakis, "Recurrent Solar Jets Active Regions," Astronomy \& Astrophysics, Vol. 512, 2010, pp. 1-4. doi:10.1038/377203a0

[21] M. S. Miesch, A. S. Brun, M. Derosa and J. Toomre, "Structure and Evolution of Giant Cells in Global Models of Solar Convection," Astrophysical Journal, Vol. 673, No. 1, 2008, pp. 557-575. doi:10.1086/523838

[22] H. K. Moffatt, "Magnetic Field Generation in Electrically Conducting Fluids," Cambridge University Press, Cambridge, 1978.

[23] N. O. Weiss and M. J. Thompson, "The Solar Dynamo," Space Science Reviews, Vol. 144, No. 1-4, 2009, pp. 53-66. doi:10.1007/s11214-008-9435-z
[24] M. Stix, "The Sun,” Springer-Verlag, Berlin, 1989.

[25] F. F. Chen, "Introduction to Plasma Physics," Plenum Press, New York, 1974.

[26] J. D. Huba and J. A. Fedder, "Self-Generation of Magnetic Fields by Sheared Flows in Weakly Ionized Plasmas," Physics of Fluids B, Vol. 5, No. 10, 1993, pp. 3779-3788. doi: $10.1063 / 1.860848$

[27] S. I. Braginskii, "In Reviews of Plasma Physics," Consultants Bureau, New York, 1965.

[28] L. Spitzer Jr., "Physics of Fully Ionized Gases," Interscience Publishers ING, New York, 1962.

[29] M. S. Wheatland and D. B. Melrose, "Alfvénic Fronts and the Turning-off of the Energy Release in Solar Flares," Proceedings of the Astronnomical Society of Australia, Vol. 11, 1994, pp. 25-27.

[30] E. N. Parker, "Comment on 'Current Paths in the Corona and Energy Release in Solar Flares'," Astrophysical Journal, Vol. 471, No. 1, 1996, pp. 489-496. doi:10.1086/177984

[31] T. M. Brown, J. Christensen-Dalsgarrd, W. A. Dziembowski, et al., "Inferring the Sun's Internal Angular Velocity from Observed p-Mode Frequency Splittings," Astrophysical Journal, Vol. 343, 1989, pp. 526-546. doi:10.1086/167727

[32] P. R. Goode, W. A. Dziembowski, S. G. Korzennik and E. J. Rhodes Jr., "What We Know about the Sun's Internal Rotation from Solar Oscillations," Astrophysical Journal, Vol. 367, 1991, pp. 649-657. doi:10.1086/169660

[33] M. Dikpati and P. Charbonneau, "A Babcock-Leighton Flux Transport Dynamo with Solar-Like Differential Rotation," Astrophysical Journal, Vol. 518, No. 1, 1999, pp. 508-520. doi:10.1086/307269

[34] J. G. Beck, "A Comparison of Differential Rotation Measurements," Solar Physics, Vol. 191, No. 1, 1999, pp. 47-70. doi:10.1023/A:1005226402796

[35] S. Tomczyk, J. Schou and M. J. Thompson, "Measurement of the Rotation Rate in the Deep Solar Interior," Astrophysical Journal, Vol. 448, 1995, pp. 57-60.

[36] P. Charbonneau and K. B. Macgregor, "On the Generation of Equipartition-Strength Magentic Fields by Turbulent Hydromagnetic Dynamos," Astrophysical Journal, Vol. 473, No. 1, 1996, pp. 59-62. doi:10.1086/310387

[37] J. N. Bahcall, M. H. Pinsonneault and S. Basu, "Solar Models: Current Epoch and Time Dependences, Neutrinos and Helioseismological Properties," Astrophysical Journal, Vol. 555, No. 2, 2001, pp. 990-1012. doi:10.1086/321493

[38] J. N. Bahcall and R. K. Ulrich, "Solar Models, Neutrino Experiments and Helioseismology," Reviews of Modern Physics, Vol. 60, No. 2, 1988, pp. 297-372. doi:10.1103/RevModPhys.60.297

[39] A. G. Kosovichev, "Solar Dynamo and Magnetic SelfOrganization," The Astronomy and Astrophysics Decadal Survey, Science White Papers, Vol. 160, 2010, pp. 1-8. 